\title{
Downscaling reveals diverse effects of anthropogenic climate warming on the potential for local environments to support malaria transmission
}

\author{
Krijn P. Paaijmans • Justine I. Blanford • \\ Robert G. Crane • Michael E. Mann • Liang Ning • \\ Kathleen V. Schreiber • Matthew B. Thomas
}

Received: 19 January 2014 / Accepted: 31 May 2014 / Published online: 19 June 2014

(C) The Author(s) 2014. This article is published with open access at Springerlink.com

\begin{abstract}
The potential impact of climate warming on patterns of malaria transmission has been the subject of keen scientific and policy debate. Standard climate models (GCMs) characterize climate change at relatively coarse spatial and temporal scales. However, malaria parasites and the mosquito vectors respond to diurnal variations in conditions at very local scales. Here we bridge this gap by downscaling a series of GCMs to provide high-resolution temperature data for four different sites and show that although outputs from both the GCM
\end{abstract}

Electronic supplementary material The online version of this article (doi:10.1007/s10584-014-1172-6) contains supplementary material, which is available to authorized users.

K. P. Paaijmans · M. B. Thomas $(\bowtie)$

Center for Infectious Disease Dynamics and Department of Entomology, The Pennsylvania State University, University Park, PA 16802, USA

e-mail: mbt13@psu.edu

K. P. Paaijmans

Barcelona Centre for International Health Research (CRESIB, Hospital Clínic-Universitat de Barcelona), Barcelona, Spain

J. I. Blanford

GeoVISTA Center, Department of Geography, The Pennsylvania State University, University Park, PA 16802, USA

R. G. Crane

AESEDA, Department of Geography, The Pennsylvania State University, University Park, PA 16802, USA

M. E. Mann · L. Ning

Department of Meteorology and Earth and Environmental Systems Institute, The Pennsylvania State University, University Park, PA 16802, USA

L. Ning

Northeast Climate Science Center, Department of Geosciences, University of Massachusetts - Amherst, Amherst, MA 01003, USA

K. V. Schreiber

Department of Geography, Millersville University of Pennsylvania, Millersville, PA 17551, USA 
and the downscaled models predict diverse but qualitatively similar effects of warming on the potential for adult mosquitoes to transmit malaria, the predicted magnitude of change differs markedly between the different model approaches. Raw GCM model outputs underestimate the effects of climate warming at both hot (3-fold) and cold (8-12 fold) extremes, and overestimate (3-fold) the change under intermediate conditions. Thus, downscaling could add important insights to the standard application of coarse-scale GCMs for biophysical processes driven strongly by local microclimatic conditions.

\section{Introduction}

Several studies predict rising global temperatures to increase the burden of malaria (Martens et al. 1999; Pascual et al. 2006). Others suggest no net change (Rogers and Randolph 2000), or even a decline in disease prevalence irrespective of changes in environmental conditions (Gething et al. 2010). These contrasting results reflect, in part, the complexity of the disease and the fact that parasite transmission by mosquito vectors is influenced by a range of intrinsic and extrinsic factors (Lafferty 2009). However, uncertainty also remains because analyses are often conducted across different scales, with inconsistencies in matching appropriate biological models of transmission with sufficiently resolved environmental data (Hay et al. 2006; Parham et al. 2012; Blanford et al. 2013).

Many models of malaria transmission characterize the environment using measures such as mean monthly temperature (Guerra et al. 2008; Rogers and Randolph 2000; Parham et al. 2012; Alonso et al. 2011). Yet other works have shown that essential components of mosquito and parasite biology are strongly affected by patterns of daily temperature fluctuation in addition to mean conditions (Paaijmans et al. 2009, 2010). Climate model (coupled Atmosphere-ocean General Circulation Model) simulation data are available and can be used for projecting potential climate change impacts on biophysical processes (Solomon et al. 2007; World Climate Research Programme). These General Circulation Model (GCM) data most confidently characterize climate changes at relatively coarse spatial and temporal scales (i.e. averaged over large regions and time through sub-continental monthly and seasonal averages). They are considerably less valid in characterizing local (point) projections on daily timescales. In addition, different climate models, even when constructed with the same basic large-scale physics and driven by the same climate change forcing, often give conflicting results owing, for example, to the way sub grid-scale processes such as atmospheric turbulence and cloud cover are represented (Meehl et al. 2007).

To project future climate change impacts at local levels, it is necessary to downscale coarsescale GCM projections to higher spatial and temporal resolutions. Downscaling can potentially reduce artificial differences in responses by, in effect, using empirical relationships between scales to bypass the GCMs' parameterizations of sub grid-scale processes (Hewitson and Crane 2006).

Here we downscale a suite of GCM simulations to derive site-specific predictions for the effects of climate warming on the potential for mosquitoes to transmit malaria in Kenya. We follow a recent study (Blanford et al. 2013) in selecting four locations that capture the broad environmental distribution of malaria, including cool upland locations (Kericho and Kitale), a warmer lower altitude site (Kisumu), and a hot savannah-like environment (Garrisa) (see Blanford et al. (2013) for full description of sites). The inclusion of Kericho is also of particular interest as this location has been at the centre of much of the malaria-climate change debate in recent years (Hay et al. 2002; Pascual et al. 2006; Chaves and Koenraadt 2010; Omumbo et al. 2011; Stern et al. 2011). 


\section{Methods}

\subsection{Climatic data}

For each of the four locations in Kenya we developed daily downscaled temperature projections by adapting an established empirical downscaling procedure (Hewitson and Crane 2006), utilizing GCM and NCEP (National Center for Environmental Prediction) Reanalysis data, as well as meteorological station observations for daily maximum and minimum temperature at each location. The station observations come from the U.S. National Climate Data Center Global Surface Summary of the Day (GSOD) data. These are obtained through the WMO World Weather Watch program and are derived from the Integrated Surface Data, which includes observations from the USAF Climatology Center. We set a minimum of four observations a day for the data to be included, and the data set is quality controlled for random errors. ${ }^{1}$

The NCEP reanalysis data are re-gridded to a nominal $2^{\circ} \times 2^{\circ}$ hexagonal grid centered on each target meteorological station, and the data from the 19 hexagonal cells centered on and surrounding the station are used to train a separate Self-Organizing Map (SOM) for each location. The SOM groups days with similar large-scale atmospheric characteristics based on the NCEP surface air temperature and specific and relative humidities at $850 \mathrm{mb}$, and the sine of the Julian day. The SOM is analogous to a non-linear fuzzy clustering algorithm, where each node in the SOM represents a group of similar days. The observed temperatures at the target location (one of the four meteorological stations) are then used to construct a cumulative frequency distribution (CFD) of temperatures for each node. The days from the present (19812000) and future (2046-2065) GCM simulations are mapped to the SOMs and a paired maximum and minimum temperature randomly selected from the appropriate CFD. The result is an empirical transfer function that maps local temperature to the larger scale dynamical forcing in a manner that also accounts for local effects - such as topography - thus producing site-specific, diurnally-resolved climate projections. Full Methods, model validation and any associated references are available in the online version of the paper.

\subsection{Malaria transmission potential}

We represent malaria transmission using a model for Lifetime Transmission Potential $(V)$, which describes the total contribution of a single mosquito over its lifetime to the transmission potential of the whole mosquito population:

$$
V=a^{2} b c \mathrm{e}^{-g n} / g^{2}
$$

where $a$ is the daily biting rate of the vector, $b c$ vector competence, $g$ the daily probability of mortality of the adult vector and $n$ the extrinsic incubation period of the parasites within the vector (Smith and McKenzie 2004). The more comprehensive Vectorial Capacity model describes the transmission potential of a mosquito population and so requires estimates of the equilibrium mosquito density per human (MacDonald 1957). While the vector:host ratio is temperature-sensitive (Mordecai et al. 2013; Parham and Michael 2010; Parham et al. 2012), numerous other factors also affect mosquito density, including rainfall (Koenraadt et al. 2004), access to permanent water bodies (van Lieshout et al. 2004; Walker and Lynch 2007), and mosquito control operations (Fillinger et al. 2009). Selecting Lifetime Transmission Potential

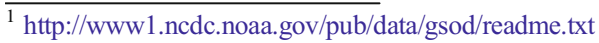


enables us to ignore these factors and examine more specifically how changes in temperature will affect the probability of malaria transmission, assuming infectious mosquitoes are present in the environment. Thus, we use Lifetime Transmission Potential as a metric to describe relative change in the suitability of the thermal environment for adult mosquitoes to transmit malaria, and not a measure of change in absolute risk (c.f. van Lieshout et al. (2004) who use a similar metric of Transmission Potential as an indicator of relative changes in transmission risk due to environmental change).

Each of these traits exhibits a strongly non-linear relationship with temperature, which we describe using thermal performance curves (reaction norms) from a recent comprehensive analysis of the temperature sensitivities of malaria mosquito and Plasmodium falciparum life history traits (Mordecai et al. 2013):

$$
\begin{gathered}
a=0.000203 T(T-11.7)(42.3-T)^{1 / 2} \\
b c=\left(-0.54 T^{2}+25.2 T-206\right) / 100 \\
g=-\ln \left(-0.000828 T^{2}+0.0367 T+0.522\right)
\end{gathered}
$$

and

$$
n=0.000111 T(T-14.7)(34.4-T)^{1 / 2} .
$$

Mean temperatures were calculated as $\left(\mathrm{T}_{\min }+\mathrm{T}_{\max }\right) / 2$. We used a standard method for generating realistic diurnal temperature variations (Parton and Logan 1981). Temperature was modelled at $1 h$ intervals using the historic and the forecast minimum and maximum temperatures of each GCM (both raw and downscaled data), assuming a day length of $12 h$. For parameters $a$ and $n$ we captured the effects of temperature variation using rate summation, whereby $a$ and $n$ were calculated at hourly intervals, and the rates summed over a day (Paaijmans et al. 2009). For $g$ and $b c$, which are described by probabilities, it is not appropriate to use rate summation so we based estimates on the mean monthly temperatures generated by the diurnal temperature models.

\section{Results}

In Fig. 1 we present the raw GCM output and the Downscaled (DS) estimates for Kisumu as an illustrative case (see Supplementary Information for the other sites and the details of individual GCMs). We present GCM and DS estimates for recent historic conditions (19812000 ) and projections to the mid-21st century (2046-2065) based on the SRES A2 emissions scenario (Nakicenovic et al. 2000). Using ensemble means (i.e. the means from the ensemble of the individual climate models) for the representative 20 -year periods, the downscaling of the model projections predict increases in future mean annual temperature of 1.9, 1.9, 1.8 and $1.9^{\circ} \mathrm{C}$ for Kericho, Kitale, Kisumu and Garrisa, respectively (Fig. 2, Table S3). They also predict small decreases in the average diurnal temperature range $\left(0.4,0.3,0.4\right.$ and $0.1{ }^{\circ} \mathrm{C}$ for Kericho, Kitale, Kisumu, and Garrisa, respectively) resulting from greater increases in minimum nighttime temperatures relative to maximum daytime temperatures (Fig. 2, Table S3). The ensemble means from the raw GCM output predict marginally lower increases in mean annual temperature (range $1.7-1.8^{\circ} \mathrm{C}$ ), but slightly larger decreases in DTR $(0.6,0.6,0.5$ and 0.4 for Kericho, Kitale, Kisumu and Garissa, respectively). The similarity in the ensemble mean changes in the raw GCM projections for the four sites simply reflects the fact that the 


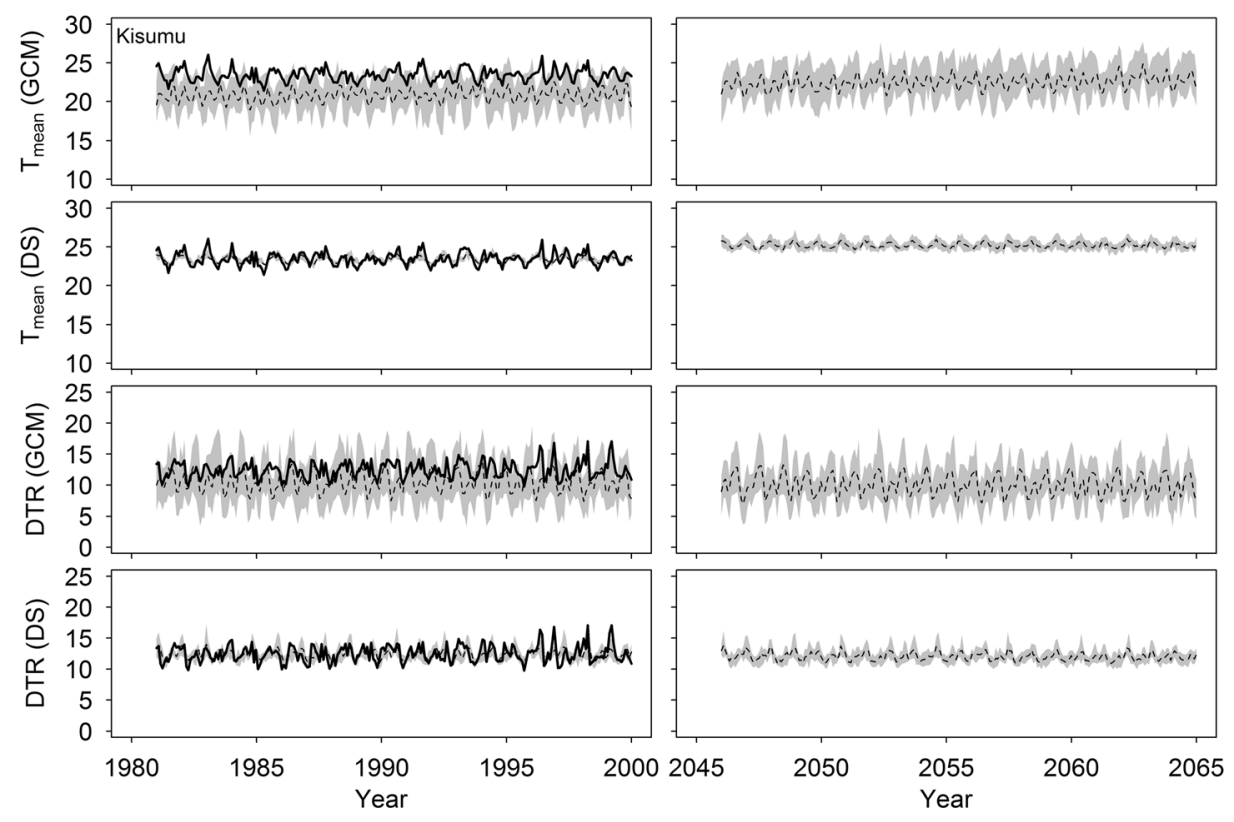

Fig. 1 Mean temperature and diurnal temperature range $(D T R)$ as estimated by the raw GCMs or the downscaled $(D S)$ models, for recent historic and future climates for Kisumu in western Kenya (outputs for the other 3 study sites are presented in Suppl. Info). The grey shading indicates the range outputs from individual climate models. The dotted black line represents the average from the ensemble of models, the solid black line the available weather station data recorded at Kisumu for a subset of the historic time series

four locations are all within one grid cell for lower resolution GCMs. The similarity in the downscaled results is potentially more interesting, but may simply be coincidental. Many more locations would need to be assessed before we could conclude anything about regional scale temperature change.

Although the raw GCM output and DS estimates predict similar increases in mean temperature, the actual raw GCM temperatures themselves differ markedly between models (Fig. 2, Table S3). The downscaled series show less variance (i.e. reduced uncertainty) than the raw GCM grid point series, smaller differences between GCMs, and much closer match to the empirical meteorological data (Fig. 1, and see the supporting online material). Furthermore, the nature of the differences between modeling approaches differs between sites. For Kericho, for example, the DS model estimates predict current and future mean annual temperatures of 17.5 and $19.5^{\circ} \mathrm{C}$, respectively, while the raw GCM outputs predict equivalent means of 20.6 and $22.3^{\circ} \mathrm{C}$. For Kisumu, on the other hand, the DS estimates predict current and future mean temperatures of 23.4 and $25.2^{\circ} \mathrm{C}$, respectively, while the raw GCMs predict 20.8 and $22.5^{\circ} \mathrm{C}$. Thus, raw GCM output can both under- and overestimate temperatures relative to downscaled GCM results.

With the climate data in hand we now explore the implications for malaria transmission. We focus on the influence of outdoor temperatures. This approach follows nearly all malariatemperature research published to date. We acknowledge that adult mosquitoes can rest indoors as well as outdoors (Alonso et al. 2011; Blanford et al. 2013). However, individual mosquito species tend to exhibit different extents of endophily and there is growing evidence that current vector control tools, such as indoor residual insecticide sprays and insecticide treated nets, are selecting for outdoor biting and resting (Govella and Ferguson 2012). 

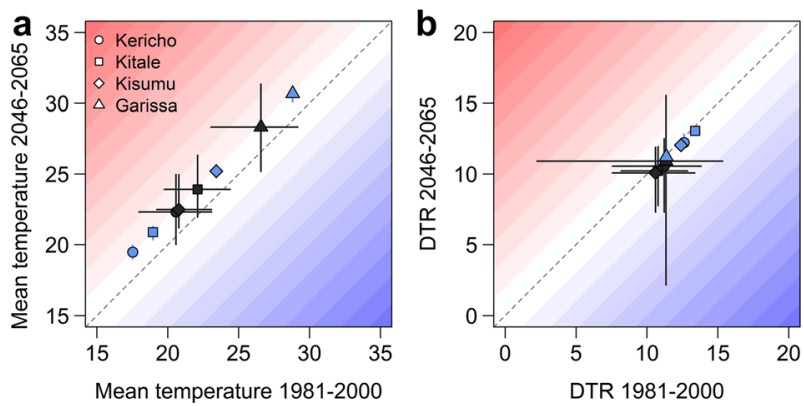

Fig. 2 Scatter plot of (a) mean temperature and (b) DTR for present day (x-axis) vs future (Y-axis) as predicted by GCMs (black symbols) and downscaled climate models (blue symbols) for 4 sites across Kenya. Data points represent 20-years average monthly temperature from the ensemble of individual models, the bars represent the range between the lowest and highest average monthly temperature predicted by an individual model. Data above dashed grey diagonal line reflect an increase in future temperatures, those below the line a decrease. Colored contours indicate increases (red) or decreases (blue) of $2{ }^{\circ} \mathrm{C}$ increments to facilitate comparisons (contours read vertically)

We first consider the effects of temperature on the Lifetime Transmission Potential $(V)$ following the standard approach using mean monthly temperatures only (Table 1). For the raw GCMs, climate warming is predicted to cause a relative increase in mean malaria transmission potential in Kericho $(+72 \%)$, Kitale $(+43 \%)$ and Kisumu $(+81 \%)$ but a modest reduction in Garissa $(-18 \%)$. Variation amongst the raw GCMs creates a broad range of potential $V$ 's around these ensemble means. The DS model results predict similar directional changes at each site but with greater magnitudes (Table 1), with relative changes in the ensemble mean $V$ values ranging from $-48 \%$ (Garissa) to $c .+1,800 \%$ (Kericho).

We next add-in the effects of daily temperature variation. The integration of daily temperature range (DTR) alters the baseline estimates of transmission potential for both the GCM and DS approaches (Table 1). With the raw GCMs (but including DTR), the prospective changes in mean temperatures alter $V$ 's by $c .+78,+46,+88$ and $-20 \%$ for Kericho, Kitale, Kisumu and Garissa, respectively. With the DS model outputs, the equivalent increases in $V$ 's are much greater for Kericho and Kitale ( +899 and $+375 \%$, respectively), slightly less for Kisumu (+ $24 \%$ ) and show a greater net reduction for Garissa (-58\%) (Table 1). Comparing these DS model outputs with the raw GCM outputs based on mean temperatures alone reveals that the standard modelling approach underestimates the effects of climate change at both hot (c.3fold) and cold extremes (c.8-12-fold), and overestimates the change under intermediate conditions (c. 3-fold) (Fig. 3).

\section{Discussion}

Our results show individual GCMs to produce highly divergent estimates of mean temperatures and daily temperature range. These in turn generate a wide range of possible current and future values of mosquito lifetime transmission potential. With such variation, the raw GCM results provide only limited insight into possible effects of climate change on malaria transmission. As noted earlier, the variation among raw GCM results is due in large part to the way different models treat sub-grid-scale processes, such as convection, cloud cover and precipitation. Multiple comparison studies indicate that the multi-model (ensemble) mean is usually more valid than any individual model (Cai et al. 2009). Even so, the ensemble means of the 
Table 1 Lifetime transmission potential $V$ as estimated by the mean temperature and diurnal temperature range (DTR) given by the raw GCMs or the downscaled (DS) models (see Table S3), for recent historic (1981-2000) and future climates (2046-2065), for 4 sites across Kenya

\begin{tabular}{clllll}
\hline & $1981-2000$ & & & $2046-2065$ & \\
\cline { 2 - 3 } \cline { 5 - 6 } & GCM & DS & & GCM & DS \\
\hline Kericho & & & & \\
Mean & $1.35 \pm 0.03(0.14-2.95)$ & $0.02 \pm 0.01(0.01-0.03)$ & $2.32 \pm 0.03(0.84-3.84)$ & $0.39 \pm 0.01(0.21-0.59)$ \\
DTR & $0.97 \pm 0.02(0.11-2.19)$ & $0.03 \pm 0.01(0.02-0.04)$ & $1.72 \pm 0.02(0.6-2.9)$ & $0.28 \pm 0.01(0.16-0.41)$ \\
Kitale & & & & & \\
Mean & $2.22 \pm 0.03(0.6-3.76)$ & $0.22 \pm 0.01(0.19-0.25)$ & $3.17 \pm 0.03(2.06-3.86)$ & $1.19 \pm 0.01(0.83-1.5)$ \\
DTR & $1.65 \pm 0.02(0.43-2.86)$ & $0.17 \pm 0.01(0.15-0.2)$ & $2.4 \pm 0.02(1.54-2.99)$ & $0.82 \pm 0.01(0.58-1.04)$ \\
Kisumu & & & & \\
Mean & $1.34 \pm 0.03(0.55-2.95)$ & $3.3 \pm 0.01(3.17-3.36)$ & $2.42 \pm 0.03(1.61-3.84)$ & $4.06 \pm 0.01(4-4.09)$ \\
DTR & $0.97 \pm 0.02(0.39-2.19)$ & $2.36 \pm 0.01(2.3-2.4)$ & & $1.82 \pm 0.02(1.13-2.9)$ & $2.9 \pm 0.01(2.84-2.97)$ \\
Garissa & & & & \\
Mean & $3.25 \pm 0.03(2.23-3.98)$ & $2.54 \pm 0.01(2.42-2.65)$ & $2.66 \pm 0.03(1.04-3.76)$ & $1.32 \pm 0.01(1.02-1.68)$ \\
DTR & $2.27 \pm 0.02(1.04-3.71)$ & $1.56 \pm 0.01(1.42-1.69)$ & & $1.82 \pm 0.02(0.47-2.89)$ & $0.66 \pm 0.01(0.49-0.91)$ \\
\hline
\end{tabular}

Data represent 20-year means from the ensemble of individual models, and the standard error of the mean. Values between parentheses are the lowest and highest average value reported by any model

raw GCMs provide a relatively poor description of the prevailing temperature conditions at the different sites (see Fig. 1 and online material). These findings resonate with recent studies suggesting that species distribution models based on fine-scale climate data may provide markedly different estimates of climate-change impacts than coarse-scale models (Franklin et al. 2013). GCMs utilize data averaged over grid cells ranging up to $4^{\circ}$ latitude $\times 5^{\circ}$ longitude. While GCMs are able to incorporate the effects of topographic variability on the large-scale dynamics of the atmosphere, this resolution is too coarse to resolve the topographically controlled climate variation that occurs between adjacent sites such as lowland Kisumu and highland Kericho.

The more finely tuned DS approach predicts large increases in future transmission potential in the cooler sites, Kericho and Kitale, but a reduction at the warmest site, Garissa. These results support the potential for complex 'two-tailed' effects of warming (Lafferty 2009), depending on the background conditions. Garissa is already at or beyond the temperature optimum for transmission (Mordecai et al. 2013), so any further increase in temperature is expected to reduce transmission potential. On the other hand, the strongly non-linear influence of temperature on transmission biology under cool conditions leads to marked increases in the $V ' s$ in the highland sites (though it should be noted that the values of lifetime transmission potential for Kericho and Kitale remain low in absolute terms). The predicted increase in transmission potential in Kisumu is less using the DS model outputs than the raw GCMs. This site is characterized by relatively consistent, year-round transmission (Blanford et al. 2013) and so even modest increases in transmission potential could translate into measurable changes in intrinsic disease risk.

As indicated previously, we use Lifetime Transmission Potential as a metric to describe relative change in the suitability of the environment for adult mosquitoes to transmit malaria. The approach follows that of others (e.g. van Lieshout et al. 2004; Weiss et al. 2014) in using a subset of all possible parameters to define relative changes in transmission suitability. Further, 


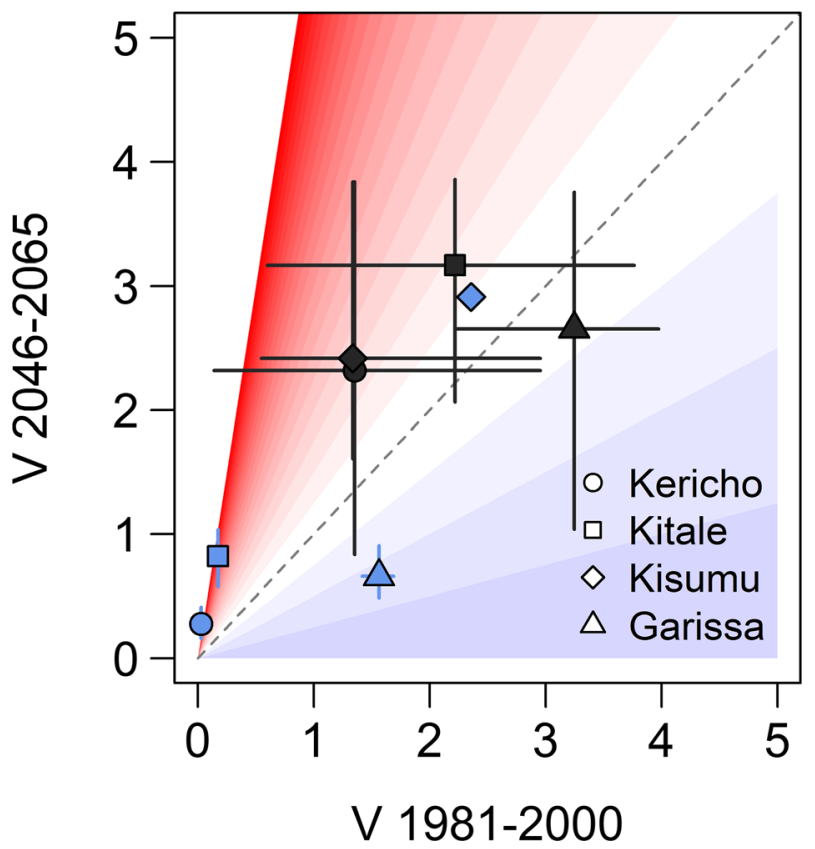

Fig. 3 Scatter plot of Lifetime Transmission Potential $(V)$ for present day (x-axis) vs future (Y-axis) as predicted by raw GCMs based on mean temperatures (black symbols) and downscaled climate models based on DTRs (blue symbols) for 4 sites across Kenya. Data points represent 20 -years average from the ensemble of models, the bars represent the range between lowest and highest average predicted by an individual model. Data above the dashed grey diagonal line indicate an increase in $V$ as a result of warming, while data below the line indicate a decrease. Colored contours indicate increases (red) or decreases (blue) in $V$ at $25 \%$ intervals (contours read vertically). The last red contour line represents a $500 \%$ increase

we consider changes in annual conditions, which overlooks potentially important effects during specific transmission seasons. Accordingly, our study is illustrative and we caution against interpreting our results as measures of changes in absolute disease risk; our basic aim is to highlight the potential for downscaling to add value to coarse scale GCM outputs (e.g. Caminade et al. 2014).

Moreover, common to nearly all climate-mosquito-malaria modeling investigations the mosquito and parasite parameter values we use to explore transmission derive largely from lab-based studies. A recent study suggested the potential for local adaptation amongst vectors and parasites creating the possibility for divergent effects of temperature due to "genotype $\times$ genotype $\times$ environment" interactions (Sternberg and Thomas 2014). However, there are few field-derived data on the temperature dependency of mosquito or parasite traits and virtually no data on malaria and mosquito local adaptation, making it difficult to explore the possible significance for transmission or control.

These caveats notwithstanding, use of downscaling techniques to identify local effects of climate warming on malaria transmission suitability could have important implications for development of local adaptation and mitigation strategies in malaria-affected areas. More generally, a growing number of studies now highlight the need to evaluate the impacts of climate change from the perspective of microclimate rather than macroclimate (De Frenne et al. 2013; Potter et al. 2013; Bakken and Angilletta 2014). Similarly, our results caution against the use of raw GCMs to explore impacts of climate change on biophysical processes 
that, like malaria transmission, exhibit considerable spatial heterogeneity and a dependence on high temporal variability in temperature.

Acknowledgments This work was supported by the NSF-EID program (grant no. EF-0914384).

Open Access This article is distributed under the terms of the Creative Commons Attribution License which permits any use, distribution, and reproduction in any medium, provided the original author(s) and the source are credited.

\section{References}

Alonso D, Bouma MJ, Pascual M (2011) Epidemic malaria and warmer temperatures in recent decades in an East African highland. Proc R Soc B 278(1712):1661-1669. doi:10.1098/rspb.2010.2020

Bakken GS, Angilletta MJ (2014) How to avoid errors when quantifying thermal environments. Funct Ecol 28 : 96-107

Blanford JI, Blanford S, Crane RG, Mann ME, Paaijmans KP, Schreiber KV, Thomas MB (2013) Implications of temperature variation for malaria parasite development across Africa. Sci Rep 3:1300

Cai XM, Wang DB, Zhu TJ, Ringler C (2009) Assessing the regional variability of GCM simulations. Geo Res Letters 36. doi:10.1029/2008g1036443

Caminade C, Kovats S, Rocklov J, Tompkins AM, Morse AP, Colón-González FJ, Stenlund H, Martens P, Lloyd SJ (2014) Proc Natl Acad Sci U S A 111:3286-3291

Chaves LF, Koenraadt CJM (2010) Climate change and highland malaria: fresh air for a hot debate. Q Rev Biol 85(1):27-55

Fillinger U, Ndenga B, Githeko A, Lindsay SW (2009) Integrated malaria vector control with microbial larvicides and insecticide-treated nets in western Kenya: a controlled trial. Bull WHO 87:655-665

Franklin J, Davis FW, Ikegami M, Syphard AD, Flint LE, Flint AL, Hannah L (2013) Modeling plant species distributions under future climates: how fine scale do climate projections need to be? Glob Chang Biol 19(2): 473-483. doi:10.1111/gcb.12051

Frenne D et al (2013) Microclimate moderates plant responses to macroclimate warming. Proc Natl Acad Sci U S A 110:18561-18565

Gething PW, Smith DL, Patil AP, Tatem AJ, Snow RW, Hay SI (2010) Climate change and the global malaria recession. Nature 465(7296):342-346. doi:10.1038/nature09098

Govella NJ, Ferguson H (2012) Why use of interventions targeting outdoor biting mosquitoes will be necessary to achieve malaria elimination. Front Physiol 3:199-199

Guerra CA, Gikandi PW, Tatem AJ, Noor AM, Smith DL, Hay SI, Snow RW (2008) The limits and intensity of Plasmodium falciparum transmission: Implications for malaria control and elimination worldwide. PLoS Med 5 (2):300-311. doi:e38

Hay SI, Cox J, Rogers DJ, Randolph SE, Stern DI, Shanks GD, Myers MF, Snow RW (2002) Climate change and the resurgence of malaria in the East African highlands. Nature 415:905-909

Hay SI, Tatem AJ, Guerra CA, Snow RW (2006) Population at malaria risk in Africa: 2005, 2015 and 2030. Foresight studies, Department of Trade \& Industry, UK

Hewitson BC, Crane RG (2006) Consensus between GCM climate change projections with empirical downscaling: precipitation downscaling over South Africa. Int J Clim 26(10):1315-1337

Koenraadt CJM, Githeko AK, Takken W (2004) The effects of rainfall and evapotranspiration on the temporal dynamics of Anopheles gambiae s.s. and Anopheles arabiensis in a Kenyan village. Acta Trop 90:141-153

Lafferty KD (2009) The ecology of climate change and infectious diseases. Ecology 90(4):888-900. doi:10. 1890/08-0079.1

MacDonald G (1957) The epidemiology and control of malaria. Oxford University Press, London

Martens P, Kovats RS, Nijhof S, de Vries P, Livermore MTJ, Bradley DJ, Cox J, McMichael AJ (1999) Climate change and future populations at risk of malaria. Global Environ Chang 9:S89-S107

Meehl GA, Tebaldi C, Teng H, Peterson TC (2007) Current and future US weather extremes and El Nino. Geo Res Lett 34(20):6

Mordecai EA, Paaijmans KP, Johnson LR, Balzer C, Ben-Horin T, de Moor E, McNally A, Pawar S, Ryan SJ, Smith TC, Lafferty KD (2013) Optimal temperature for malaria transmission is dramatically lower than previously predicted. Ecol Lett 16:22-30 
Nakicenovic N, Alcamo J, Davis G, De Vries B, Fenhann J, Gaffin S, Gregory K, Grübler A, Jung TY, Kram T, La Rovere EL, Michaelis L, Mori S, Morita T, Pepper W, Pitcher H, Price L, Riahi K, Roehrl A, Rogner HH, Sankovski A, Schlesinger M, Shukla P, Smith S, Swart R, Van Rooijen S, Victor N, Dadi Z (2000) Special report on emissions scenarios: a special report of working group III of the Intergovernmental panel on climate change. Cambridge, UK

Omumbo J, Lyon B, Waweru S, Connor S, Thomson M (2011) Raised temperatures over the Kericho tea estates: revisiting the climate in the East African highlands malaria debate. Malar J 10(1):12

Paaijmans KP, Read AF, Thomas MB (2009) Understanding the link between malaria risk and climate. Proc Natl Acad Sci U S A 106:13844-13849

Paaijmans KP, Blanford S, Bell AS, Blanford JI, Read AF, Thomas MB (2010) Influence of climate on malaria transmission depends on daily temperature variation. Proc Natl Acad Sci U S A 107:15135-15139. doi:10. 1073/pnas.1006422107

Parham PE, Michael E (2010) Modeling the effects of weather and climate change on malaria transmission. Environ Health Perspect 118(5):620-626. doi:10.1289/ehp.0901256

Parham P, Pople D, Christiansen-Jucht C, Lindsay S, Hinsley W, Michael E (2012) Modeling the role of environmental variables on the population dynamics of the malaria vector Anopheles gambiae sensu stricto. Malar J 11(1):271

Parton WJ, Logan JA (1981) A model for diurnal variation in soil and air temperature. Agric Met 23:205-216

Pascual M, Ahumada JA, Chaves LF, Rodó X, Bouma M (2006) Malaria resurgence in the East African highlands: temperature trends revisited. Proc Natl Acad Sci U S A 103(15):5829-5834

Potter KA, Woods HA, Pincebourde S (2013) Microclimatic challenges in global change biology. Global Chang Biol 19:2932-2939

Rogers DJ, Randolph SE (2000) The global spread of malaria in a future, warmer world. Science 289 (8 September):1763-1766

Smith DL, McKenzie FE (2004) Statics and dynamics of malaria infection in Anopheles mosquitoes. Malar J 3: 13

Solomon S, Qin D, Manning M, Alley RB, Berntsen T, Bindoff NL, Chen Z, Chidthaisong A, Gregory JM, Hegerl GC, Heimann M, Hewitson B, Hoskins BJ, Joos F, Jouzel J, Kattsov V, Lohmann U, Matsuno T, Molina M, Nicholls N, Overpeck J, Raga G, Ramaswamy V, Ren J, Rusticucci M, Somerville R, Stocker TF, Whetton P, Wood RA, Wratt D (2007) Contribution of working group I to the fourth assessment report of the intergovernmental panel on climate change. Climate change 2007: the physical science basis. Cambridge, UK

Stern DI, Gething PW, Kabaria CW, Temperley WH, Noor AM, Okiro EA, Shanks GD, Snow RW, Hay SI (2011) Temperature and malaria trends in Highland East Africa. PLoS One 6(9):e24524. doi:10.1371/ journal.pone.0024524

Sternberg ED, Thomas MB (2014) Local adaptation to temperature and the implications for vector-borne diseases. Trends Parasitol 30:115-122

van Lieshout M, Kovats RS, Livermore MTJ, Martens P (2004) Climate change and malaria: analysis of the SRES climate and socio-economic scenarios. Global Environ Chang 14:87-99

Walker K, Lynch M (2007) Contributions of Anopheles larval control to malaria suppression in tropical Africa: review of achievements and potential. Med Vet Entomol 21:2-21

Weiss DJ, Bhatt S, Mappin B, Van Boeckel TP, Smith DL, Hay SI, Gething PW (2014) Air temperature suitability for Plasmodium falciparum malaria transmission in Africa 2000-2012: a high-resolution spatiotemporal prediction. Malar J 13:171 